\title{
Liderazgo ético para el fortalecimiento de una cultura organizacional en las Pymes
}

\section{Ethical leadership to strengthen an Organizational Culture in SMEs}

INFORMACIÓN DEL ARTÍCULO

Fecha de recepción: 25 de Junio de 2021.

Fecha de aceptación: 24 de Octubre de 2021.

${ }^{1}$ Doctor en Administración Estratégica de Empresas, CENTRUM PUCP Docente-investigador, Instituto Superior Tecnológico CotacachiEcuador

E-mail: sleal@institutocotacachi.edu.ec Código ORCID:

https://orcid.org/0000-0002-8448-7201

2 Maestrante en Riesgos Laborales, UNIR. Docente-investigador, Instituto Superior Tecnológico CotacachiEcuador.

E-mail: barias@institutocotacachi.edu.ec Código ORCID:

https://orcid.org/0000-0002-8336-7945

CITACIÓN: Leal Paredes, M.S., \& Arias Ibarra, B.P. (2021). Liderazgo ético para el fortalecimiento de una cultura organizacional en las Pymes. Podium, 40, 21-40.

doi:10.31095/podium.2021.40.2

\section{ENLACE DOI:}

http://dx.doi.org/10.31095/podium.202 1.40 .2

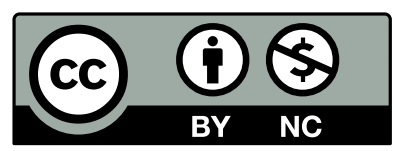

Marlon Santiago Leal Paredes ${ }^{1}$, Byron Patricio Arias Ibarra ${ }^{2}$

\section{Resumen}

El objetivo de la investigación es determinar el impacto del liderazgo ético sobre la cultura organizacional en gerencia de las pymes de Imbabura. Para alcanzar este propósito se identificaron los rasgos de la personalidad de los líderes éticos y las características de la cultura organizacional. La investigación se desarrolló con un enfoque cuantitativo y descriptivo, con un estudio explicativo y un diseño no experimental de corte transversal. Para la recolección de los datos se diseñaron los cuestionarios del liderazgo ético y de la cultura organizacional. Los resultados demostraron que los empleados de las pymes perciben en los líderes de las pymes los rasgos de la personalidad del liderazgo ético y que éstos rasgos tienen una correlación positiva y directamente proporcional sobre las características de la cultura organizacional. Se concluye que el liderazgo ético fortalece la cultura organizacional en las pymes.

\section{Palabras Clave:}

Liderazgo, Ética, Pymes, Cultura, Organización, Desempeño, Cultura Organizacional.

Clasificación JEL: M1, M12, M14.

\begin{abstract}
The objective of the research is to determine the impact of ethical leadership on the organizational culture in management of SMEs in Imbabura. To achieve this purpose, the personality traits of the ethical leaders and the characteristics of the organizational culture were identified. The research was developed with a quantitative and descriptive approach, with an explanatory study and a non-experimental cross-sectional design. For data collection, the ethical leadership and organizational culture questionnaires were designed. The results showed that SME employees perceive the personality traits of ethical leadership in SME leaders and that these traits have a positive and directly proportional correlation on the characteristics of the organizational culture. It is concluded that ethical leadership strengthens the organizational culture in SMEs.
\end{abstract}

Keywords:

Leadership, Ethics, SMEs, Culture, Organization, Performance, Organizational Culture.

JEL Classification: M1, M12, M14. 


\section{Introducción}

En el entorno empresarial contemporáneo la situación subyacente refleja la necesidad de muchas empresas de reevaluar su perspicacia estratégica para el desarrollo de una gestión que permita la consolidación del liderazgo ético con fines de eficiencia $y$ rentabilidad empresarial (Alshammari, Almutairi, y Fahad Thuwaini, 2015). El liderazgo ético se fundamenta en la credibilidad que los empleados sientan hacia jefe, en la tenacidad en sus actividades, en la flexibilidad para adaptarse a las circunstancias cambiantes, a la humildad en el reconocimiento de sus debilidades, cualidades y capacidades, en el profesionalismo, en la manera de hacer las cosas y en el compromiso que ejerce dándole el frente a los empleados como un ente motivador (Gómez, 2006). El liderazgo ético se define como la demostración de conductas normativamente apropiadas a través de acciones personales y relaciones interpersonales, así como la promoción de tales conductas en los seguidores a través de la comunicación bilateral, el refuerzo y la toma de decisiones (Brown, Treviño, y Harrison, 2005). Los rasgos que identifican la personalidad de los líderes éticos permiten evidenciar el buen desempeño de los empleados (Correa, Rodríguez, y Alonso, 2018).

Algunos autores señalan que el liderazgo ético puede afectar fuertemente la moral y la lealtad de los empleados, así como la cultura organizacional ya que el comportamiento del líder es determinante para la organización (Khuong y Nhu,
2015). Otros autores arguyen que el comportamiento del liderazgo ético afecta la cultura organizacional ya que su ejercicio mediante la toma de decisiones éticas influye inherentemente en las actitudes e interacciones de los empleados (Alshammari et al., 2015). En este sentido, el objetivo de este estudio es investigar el efecto que produce el liderazgo ético sobre la cultura organizacional en pequeñas y medianas empresas de Ecuador.

En términos generales, se debe enunciar que sobre la vinculación entre el liderazgo ético y la cultura organizacional en Ecuador representa un vacío dentro de los hallazgos empíricos de la comunidad científica. Un estudio realizado en Machala, identificó las prácticas de un buen liderazgo en las pequeñas y medianas empresas comerciales (Enderica et al., 2018). No obstante, algunos autores señalan que en Ecuador, los estudios sobre liderazgo son muy limitados (Espinoza-Solís y Elgoibar, 2019). Con respecto a las investigaciones sobre la cultura organizacional en Ecuador, la literatura también es escasa, sin embargo, existe un estudio sobre el diagnóstico de la cultura organizacional de una universidad de Guayaquil cuyos resultados revelaron una percepción de inconformidad en los estudiantes sobre el liderazgo, la comunicación y las relaciones como las variables que caracterizan su cultura organizacional (Llanos, 2018). Otro estudio reveló el impacto positivo de la cultura organizacional sobre un modelo de gestión (Jiménez, Pazmay, y Mancheno, 2017). 
Del mismo modo, autores recientes indican la necesidad de estudios que determinen la relación entre las características del liderazgo y la cultura organizacional. Las investigaciones realizadas sobre el liderazgo como la cultura organizacional establecen que tanto la cultura organizacional como el liderazgo pueden comportarse de manera diferente dependiendo del contexto en el que se encuentren, pero la relación entre estos dos constructos es una de las escasas investigaciones realizadas en pequeñas y medianas empresas (Palafox Soto, Ochoa Jiménez, y Jacobo Hernández, 2021). En este sentido, el objetivo de la investigación se fundamenta en determinar el impacto del liderazgo ético sobre la cultura organizacional en gerencia de las pymes de Imbabura.

\section{Revisión de literatura}

\section{Liderazgo Ético}

El liderazgo ético ha atraído una atención masiva en el siglo XXI, sin embargo, a pesar de esta vasta literatura, el conocimiento del liderazgo ético adolece de las conceptualizaciones existentes que combinen los comportamientos éticos de los líderes con las evaluaciones de los seguidores (Banks, Fischer, Gooty, y Stock, 2020) y en función del compromiso en el lugar de trabajo (Joplin, Greenbaum, Wallace, y Edwards, 2021). El liderazgo se considera como una relación de influencia que se da entre el líder y sus seguidores, mediante la cual ambas partes buscan el logro de cambios y propósitos compartidos (Daft, 2015). Los valores éticos y el comportamiento son aspectos importantes de varias teorías prominentes en la literatura sobre liderazgo (Avolio \& Gardner, 2005). De hecho, se sugiere que los líderes éticos incorporen principios morales en sus creencias, valores y comportamientos (Khuntia y Suar, 2004).

En términos generales, la conducta de las personas radica en la calidad de vida privada, pero cuando esa persona desempeña una función directiva o del ejercicio del liderazgo, sus actitudes también influyen en la organización que dirige (Gómez, 2006). Los líderes como modelos a seguir motivan el comportamiento ético al demostrar el tipo de acciones que quieren promover $\mathrm{y}$ recompensar constituyéndose como una guía informativa para comportamientos aceptables (Bedi, Alpaslan, y Green, 2016). De esta forma se debe acotar que los líderes éticos se involucran en actos y comportamientos que benefician a otros $\mathrm{y}$, al mismo tiempo, se abstienen de comportamientos que puedan causar algún daño a los demás (Kanungo, 2001).

Algunos autores definen el liderazgo ético como aquellos comportamientos e interacciones del líder que se consideran moralmente aceptables, así como la promoción de estos hacia sus seguidores a través del ejercicio del liderazgo (Correa, et al., 2018). Brown et al. (2005) explicaron que el comportamiento del líder ético se conceptualiza en función de dos componentes, el primero en el reflejo de la demostración de una conducta normativamente apropiada a través de 
acciones personales y relaciones interpersonales; y el segundo en la promoción de dicha conducta entre los seguidores a través de la comunicación bidireccional, el refuerzo y la toma de decisiones. Posteriormente, Yukl (2010) propone una concepción del liderazgo ético como un constructo que se centra en analizar éticamente dos dimensiones: la primera se fundamenta en las características personales del líder, en función de sus valores personales, el nivel de desarrollo moral y los tipos de influencia utilizados; y la segunda dimensión hace referencia a la forma en que toma decisiones y actúa, lo que involucra fines, medios y resultados, usualmente considerados en relación con los demás.

En épocas más recientes, otros autores (Ahmad y Gao, 2018) explican que un líder ético es una persona moral y un administrador moral, siendo estas dos características simultáneas en su comportamiento. La primera, es denotada por sus acciones como una persona moral y se refiere a la característica de la personalidad y la motivación altruista que demuestra su honestidad, integridad y confiabilidad. La segunda se refiere a tomar decisiones justas y equilibradas, expresar expectativas de rol alineadas con las metas organizacionales y mostrar un cuidado sincero por los seguidores (Ahmad y Gao, 2018; Leal Paredes, Salomon, y Rivera Camino, 2021). Esto significa que los líderes deben estar comprometidos a comportarse éticamente tanto en su vida personal como profesional y a hacer las cosas basándose en reglas de decisión ética. En este sentido, el ejercicio del liderazgo ético se caracteriza en su actitud de compromiso ya que un líder ético es capaz de convencer a los empleados para hacer que se sientan comprometidos con la organización, de no derrumbarse ante las dificultades dando el frente a las situaciones, de motivar a los empleados luchando por su formación; y posee la capacidad de cultivarse como una persona moralmente íntegra (Gómez, 2006).

Se ha argumentado que el liderazgo ético es importante para las organizaciones debido a sus efectos positivos en una variedad de actitudes y comportamientos de los empleados, como la satisfacción laboral, el desempeño en el rol, la participación, el comportamiento de ciudadanía organizacional, el bienestar y la creatividad (Lindblom, Kajalo, y Mitronen, 2015). Los líderes éticos brindan tareas desafiantes a sus seguidores, los alientan a ser creativos y prestan mucha atención a sus necesidades de crecimiento y desarrollo, compartiendo así características de motivación inspiradora, estimulación intelectual y líderes de consideración individualizada (Bedi et al., 2016).

Las investigaciones sobre el liderazgo ético, más allá de demostrar la importancia del tema como medio para prevenir la ocurrencia de actos no éticos en las organizaciones, han permitido verlo por los rasgos que identifican su personalidad como una forma de fortalecer aspectos tan importantes como el desempeño de los empleados, las 
culturas organizacionales éticas y el clima laboral (Correa et al., 2018). En tal sentido, es pertinente acotar que los autores Mihelic, Lipicnik, y Tekavcic (2010), agrupan los rasgos de la personalidad de los líderes éticos que se pueden resumir en los siguientes:

Considerado y reflexivo: El primer requisito que debe cumplir el líder es el compromiso con su colectivo (Rodríguez, 2008). Cuando un líder ético se compromete con los empleados se dedica con el corazón a cumplir las metas con esfuerzo, con equidad, justicia (Mihelic et al., 2010). Los líderes éticos siempre cumplen lo prometido y respetan los derechos de los empleados dando ejemplos de buen comportamiento (Gómez, 2006). Los empleados perciben la actitud de los líderes éticos que se comprometen cuando piensan en el bienestar de los demás, son humildes, honestos y directos en su trato dentro y fuera del lugar de trabajo, no discriminan a ningún empleado; $y$ asumen sus responsabilidades cumpliendo con sus deberes (Mihelic et al., 2010).

Bondadoso y respetuoso: Los autores distinguen que un líder ético actúa al servicio de los demás como empleador o supervisor, que tiene poder, control y autoridad sobre otras personas. Siempre es respetuoso con sus subordinados y escucha todos sus problemas y quejas. Un líder ético nunca es dictatorial ni arrogante en su trabajo con los demás, por el contrario, brinda apoyo a los empleados ante la ejecución de tareas laborales difíciles (Mihelic et al., 2010). Un líder ético es flexible, es capaz de entender los puntos de vista y los sentimientos de los empleados, y de sostener un dialogo sin herir o avasallar con opiniones categóricas o descalificantes (Gómez, 2006). Un gerente con cualidades de liderazgo ético se orienta al bienestar común y lucha por el bienestar de los empleados en la empresa, siendo un líder éticamente responsable (Cortina, 2007).

Valores: El liderazgo ético está estrechamente asociado con los principios, valores y normas éticas como parte integral de cualquier tipo de trabajo. Los valores son principios generales que controlan y orientan los procesos y acciones (Brown y Treviño, 2006). Son principios que en una organización regulan el comportamiento de las personas en función de lo que se considera bueno, justo o correcto (Cortina, 2007). Los valores actúan como patrones básicos de comportamiento ético de los líderes determinando su posición frente a los empleados con honestidad, respeto, solidaridad, tolerancia, entre otros (Rodríguez, 2008). Los valores éticos dentro de la organización se enfatizan y fortalecen principalmente a través de un liderazgo basado en valores que puede definirse como una relación entre líderes $\mathrm{y}$ compañeros de trabajo, basada en valores comunes internalizados sobre los que actúa el líder (Mihelic et al., 2010).

Pensamiento positivo: Un líder ético siempre piensa en positivo no desfallece cuando experimenta fallas, lo que le permite llevar a cabo sus funciones y tareas de forma apropiada (Mihelic et al., 
2010). Un gerente ético piensa positivamente ante los derechos y desarrollo técnico de los empleados dentro de una organización (Soto $\mathrm{y}$ Cárdenas, 2007), se preocupa por mantener una mentalidad global en función de todo el equipo de la organización, contribuyendo no solo con cada uno de los empleados, sino luchando por todo aquello que es bueno para toda la organización (Cortina, 2007). Los pensamientos positivos dentro del ser automática y fácilmente crean sentimientos de cooperación en la mente de los demás (Bañón, 2007).

Prudencia y perseverancia: Un líder con el hábito de ejercitar la prudencia y la determinación no se inclina a recurrir a prácticas poco éticas, incluso en momentos en que las cosas no salen según lo planeado ya que su persistencia hace referencia al esfuerzo por alcanzar metas y su búsqueda continua de tomar todos los pasos esenciales para alcanzarlas, incluso si implican gastos y riesgo personal (Mihelic et al., 2010). La prudencia es la virtud de actuar de forma justa, adecuada y con cautela; de comunicarse con los demás por medio de un lenguaje claro, literal y cauteloso y adecuado; de crear situaciones o tomar decisiones respetando los sentimientos, la vida y las libertades de aquellos quienes se puedan ver afectados por esa situación o esa decisión (Rodríguez, 2008). Un líder ético es una persona equilibrada, que no actúa impulsivamente, que analiza la situación antes de tomar las decisiones para garantizar a los miembros de la organización una mejor solución para cualquier eventualidad (Soto y Cárdenas, 2007).
Integridad: Los líderes éticos son los que muestran integridad, son honestos consigo mismos y con los demás, aprenden de los errores y están continuamente en el proceso de superación personal demostrando su responsabilidad de juzgar las decisiones importantes y se esfuerzan por equilibrar los intereses en competencia cuando se encuentran en el proceso de alcanzar las metas de la organización (Mihelic et al., 2010). Un líder ético fomenta en la organización el trabajo en equipo, con la aceptación, la tolerancia, la confianza y el apoyo de los empleados sumando fuerzas individuales (Frondizi, 2005). Los líderes éticos están dispuestos a colaborar en cualquier eventualidad que se presente dentro de una organización, hacen que el desarrollo de las tareas sea sinérgico, en un ambiente laboral sano, cálido y agradable entre los empleados alcanzando los mejores resultados para la productividad organizacional (Pacheco y Sierra, 2007).

\section{Cultura Organizacional}

La cultura organizacional es un importante constructo, sus conceptualizaciones en investigaciones recientes han revelado su complejidad y variedad (Lam, Nguyen, Le, y Tran, 2021). Un examen de las definiciones y conceptualizaciones de la cultura organizacional por diferentes investigadores revela la complejidad y variedad de este factor. Se han realizado estudios previos para evaluar algunos de los componentes de comportamiento de la cultura empresarial. Sin embargo, los instrumentos válidos y confiables utilizados para medir las 
características importantes y reconocibles de la cultura en el contexto de las empresas de alta tecnología siguen siendo limitados.

Son muchas las definiciones sobre la cultura organizacional que han surgido dentro de la comunidad científica. La cultura organizacional es el conjunto de supuestos, creencias, valores y normas que comparten los miembros de una organización (Newstrom, 2011). La cultura organizacional es un patrón de supuestos básicos aprendidos que se comparten en una organización para resolver los problemas de adaptación externa e integración interna, y que ha funcionado lo suficientemente bien como para ser considerados válidos, $\mathrm{y}$ por lo tanto, ser enseñados a nuevos miembros como la manera correcta de percibir, pensar y sentir en relación a esos problemas (Schein, 2010).

En las organizaciones, la cultura representa un conjunto de convicciones, valores y normas que comparten los empleados de una empresa, pudiendo haber sido conscientemente creada por sus miembros principales o sencillamente puede haber evolucionado en el curso del tiempo, representando un elemento clave del entorno de trabajo en el que los trabajadores desempeñan sus labores (Davis y Newstrom, 2001). En este sentido, se dice que el primer paso para conocer a una organización es conocer su cultura en la cual se comparten una serie de valores y creencias que influyen en el comportamiento de los empleados (Chiavenato, 2009).
La cultura organizacional se caracteriza por la regularidad en los comportamientos de los empleados a través del lenguaje y los rituales, por las normas como pautas de conductas y las políticas de trabajo, por los valores dominantes que comparten los empleados; y por la filosofía que se refleja en la organización sobre la manera de tratar a los empleados o clientes (Luthans, 2011). Del mismo modo, en el contexto organizacional a partir de dos dimensiones, Cameron y Quinn (2011) definen cuatro tipos de cultura en función de un modelo de valores de competencia. La primera dimensión describe la estabilidad frente a la flexibilidad haciendo referencia si la organización considera más importante el orden y el control (estabilidad) que el dinamismo y la discrecionalidad (flexibilidad); y la segunda dimensión describe la preocupación de la organización por su personal y procesos frente a la preocupación por sus clientes, competidores y el entorno. Al combinar ambas dimensiones se pueden identificar los siguientes tipos de cultura: clan, adhocrática, jerárquica y de mercado.

Por su parte, Denison (1996) establece que los sistemas gerenciales en las organizaciones reflejan las creencias, los supuestos básicos, los valores y los principios como parte de la cultura organizacional que guía el comportamiento y el sentido de permanencia de sus miembros. Posteriormente, junto a sus colaboradores, el autor establece un modelo de cultura organizacional fundamentado sobre una teoría que se relaciona con el desempeño de la 
empresa (Denison, Haaland, y Goelzer, 2003). De hecho, el autor acota que los estudios de la cultura en las organizaciones han señalado la necesidad del empoderamiento para promover la consistencia entre el comportamiento y los elementos culturales para el mejor desempeño de los empleados. En esta relación, la implicación, la consistencia, la adaptabilidad y la misión, constituyen el enfoque teórico (Denison, Hooijberg, Lane, y Lief, 2012) que permite determinar los rasgos básicos de la cultura organizacional.

Implicación: Se refiere al compromiso personal de los empleados dentro de la organización y refleja un enfoque en la dinámica interna de la empresa y en la flexibilidad (Denison, Nieminen, y Lindsey, 2012). Este rasgo de la cultura organizacional se fundamenta en tres indicadores de comportamiento. El primero se manifiesta cuando el empleado disfruta del empoderamiento, es decir, cuenta con la autoridad la iniciativa y la capacidad para administrar su propio trabajo, en consecuencia, se crea un sentido de propiedad $y$ responsabilidad del trabajador hacia la organización. El segundo se refleja cuando la empresa orienta a sus empleados hacia el trabajo en equipo valorando el trabajo cooperativo hacia metas comunes por las cuales todos los trabajadores se sienten mutuamente responsables. El tercer indicador de la cultura organizacional a través de la participación es el interés de la gerencia por el desarrollo de las capacidades de los empleados a través de la inversión para mantener la competitividad y satisfacer las necesidades comerciales actuales (Denison et al., 2012).

Consistencia: Se refiere a facilitar acciones coordinadas y promover la coherencia de los comportamientos de los empleados con los valores empresariales centrales (Denison et al., 2012). Esta característica de la cultura organizacional se refleja en tres indicadores de conducta en los empleados. El primero se hace presente cuando los empleados comparten los valores fundamentales creando un sentido de identidad y un conjunto claro de expectativas. El segundo cuando los miembros de la organización pueden llegar a un acuerdo sobre cuestiones críticas, los empleados participan cuando existen desacuerdos para encontrar soluciones en las que todos puedan ganar. El tercer indicador de la consistencia se refleja cuando la coordinación e integración de las diferentes funciones y unidades de la organización pueden trabajar bien juntas para lograr objetivos comunes. En este sentido, los empleados no tienen limitaciones en la empresa para la realización de su trabajo.

Adaptabilidad: Se trata de traducir las demandas del entorno organizacional en acciones (Denison et al., 2012). Es la capacidad de una empresa ante a las señales del mercado y de los clientes tanto internos como externos para responder a los cambios del entorno, y a reestructurarse institucionalizando conductas y procesos que les permita adaptarse (Denison et al., 2003). Esta característica de la cultura organizacional se refiere a la capacidad de los empleados para 
comprender lo que quiere el cliente, aprender nuevas habilidades y cambiar en respuesta a la demanda (Denison et al., 2012). Este rasgo se puede visualizar a través de tres indicadores de conducta organizacional. El primero se manifiesta en la capacidad de la empresa de crear cambios $o$ formas adaptables para satisfacer las necesidades cambiantes, cuando es capaz de leer el entorno empresarial, reaccionar rápidamente a las tendencias actuales y anticipar cambios futuros. El segundo cuando la organización se enfoca en el cliente anticipándose a la satisfacción de sus necesidades futuras; $y$ el tercer indicador de adaptabilidad que caracteriza la cultura organizacional se percibe a través del aprendizaje organizacional, cuando la empresa recibe, traduce e interpreta señales del entorno en oportunidades para fomentar la innovación, adquirir conocimientos y desarrollar capacidades (Denison et al., 2012).

Misión: Se refiere al sentido claro de propósito o dirección que define las metas organizacionales y los objetivos estratégicos expresados en la visión de lo que la organización quiere ser en el futuro (Denison et al., 2003). Este rasgo se puede observar en dirección e intención estratégica, metas y objetivos, y visión (Mintzberg, 2002). Las fortalezas en este rasgo indican que el personal tiene claro lo que persigue y hacia dónde va la empresa, por qué hace lo que hace y lo que consigue la empresa con ello (Denison et al., 2003). Del mismo modo, Denison et al. (2012) distinguen tres indicadores que denotan la misión como característica de la cultura organizacional.
El primero se fundamenta en la intención y dirección estratégica que se hace presente cuando las intenciones estratégicas establecidas transmiten el propósito de la organización y dejan claro cómo todos pueden contribuir y "dejar su huella" en la industria. El segundo se manifiesta a través de las metas y objetivos que se distinguen como un conjunto claro para vincular a la misión, la visión y la estrategia, y proporcionar a todos una dirección clara en su trabajo. Finalmente, el tercer indicador de la misión es la visión compartida de un estado futuro deseado dentro de la organización que encarna valores fundamentales y captura los corazones y las mentes de los empleados de la empresa, al tiempo que brinda orientación y dirección (Denison et al., 2012).

\section{Liderazgo Ético y Cultura Organizacional}

La cultura organizacional y el liderazgo son dos caras de la misma moneda (Schein, 2004). El liderazgo es una consecuencia de la cultura organizacional y esta es el resultado del ejercicio del liderazgo, es decir, el liderazgo y la cultura organizacional son dos constructos que están entrelazados, ya que uno requiere del otro (Schein, 2010). En las organizaciones, la creación de la cultura permite identificar los comportamientos adecuados para el éxito. De hecho, el ejercicio del liderazgo con altos niveles de comunicación en apoyo a la cultura de la organización constituye un ejemplo que define las reacciones de los miembros de la 
organización (Fairholm, 1994). En este sentido, la influencia que un líder puede ejercer sobre la cultura de una organización es diferente si dicho líder es el fundador de la organización, si ejerce su labor en el marco de una organización ya consolidada o si la organización atraviesa un periodo de crisis (Trice y Beyer, 1993).

Algunos autores señalan que la influencia ejercida por los líderes y el estilo en la toma de decisiones se adopta en función de la cultura organizacional y por ello se afirma que la eficiencia del liderazgo depende del impacto que causa la cultura organizacional (Avolio y Bass, 1995). Otros autores explican que se ha demostrado que el liderazgo contribuye en gran medida a la creación, transformación y mantenimiento de las culturas organizacionales debido que la influencia del liderazgo sobre la cultura organizacional se realiza a través de una serie de conductas excepcionales que han sido atribuidas en muchas ocasiones a los líderes (Molero, 2002). No obstante, la ética en los líderes refleja el comportamiento que los gerentes adoptan en su trabajo diario, en el cual se respetan los principios éticos y las normas establecidas por ellos mismos para orientar la cultura organizacional (Mintzberg, 2002).

Esto significa que el ejercicio de liderazgo, puede dar como resultado una serie de culturas organizacionales con distintos climas organizacionales (Aktouf, 2002). En las organizaciones la creación y la permanencia de la cultura influye en el comportamiento gerencial basado en las acciones y el estilo de liderazgo (Schein, 2004). El estilo de liderazgo es el producto del vínculo entre la estrategia y la cultura que afecta el desempeño en niveles organizacionales e individuales (Burke y Litwin, 1992).

Si bien es cierto que los líderes éticos poseen valores y principios estrechamente ligados a la moral que describen su comportamiento hacia el fortalecimiento de una adecuada convivencia dentro de la organización (Donawa, 2019), no es menos cierto que la cultura organizacional también aporta los valores, creencias y principios que fundamentan los sistemas gerenciales (Denison, 1996), es por ello la inferencia sobre una marcada relación entre el liderazgo ético y la cultura organizacional.

\section{Metodología}

El enfoque de la investigación se desarrolló bajo el paradigma epistemológico post-positivista ya que se utilizan teorías específicas de forma inductiva, se prueban las teorías, se operacionalizan las variables derivadas de esas teorías y se seleccionan los instrumentos adecuados para medir las variables (Creswell y Creswell, 2018). En tal sentido, la investigación tiene un enfoque cuantitativo que aplicó la lógica deductiva a través del método inductivo-deductivo, que consiste en un procedimiento que parte de unas aseveraciones teóricas y busca comprobar, refutar o falsear tales afirmaciones, deduciendo de ellas conclusiones que deben confrontarse con 
los hechos (Bernal, 2010).

Cabe notar que el estudio permitió describir la caracterización de hechos, fenómenos de grupos de personas con el propósito de establecer su estructura o comportamiento (Arias, 2012). Con ello se pretende significar que la investigación, se enmarca dentro de la tipología de investigación descriptiva que no requiere de hipótesis (Hernández, Fernández, y Baptista, 2014), pues se orienta a recolectar información sobre un grupo para determinar los comportamientos del liderazgo ético en las pymes, identificando características, formas de conducta (Méndez, 2012). El diseño fue no experimental de corte transversal o transeccional ya que la recolección de los datos se llevó a efecto en un solo momento y en un tiempo único (Hernández et al., 2014).

La población del estudio corresponde a los empleados que prestan sus servicios a las pequeñas y medianas empresas (pymes) de la provincia de Imbabura. Las pymes son definidas como unidades económicas productoras de bienes $\mathrm{y}$ servicios, dirigidas por sus propietarios, de una forma personalizada y autónoma, de pequeña dimensión en cuanto a número trabajadores y cobertura de mercado (Cardozo, Velásquez, y Rodríguez, 2012). El Instituto Nacional de Estadística y Censos (INEC, 2018) de Ecuador establece que en Imbabura existen 162 pequeñas y 58 medianas empresas con 4.040 y 4.257 empleados respectivamente. Con la aplicación de la ecuación de Sierra (2000) se calculó el tamaño de la muestra para obtener la representatividad de la población quedando constituida por 729 empleados ordinarios, de ambos sexos, diferentes edades; y profesionales y no profesionales, los mismos que fueron seleccionados a través del método de muestreo aleatorio simple.

A partir de la operacionalización de las variables en dimensiones e indicadores, se diseñó un cuestionario para la evaluación del comportamiento del liderazgo ético y otro para la cultura organizacional de 18 y 15 ítems respectivamente, sometidos a la evaluación de 7 expertos en el área de estudio. El cuestionario de liderazgo ético compuesto de seis dimensiones: considerado y reflexivo (4 ítems, por ejemplo, «Mi jefe inmediato se compromete con sus empleados»), bondadoso y respetuoso (3 ítems, por ejemplo, «Mi jefe inmediato se orienta al bienestar común»), valores (2 ítems, por ejemplo, «Mi jefe inmediato actúa con valores de honestidad, respeto, solidaridad y tolerancia», pensamiento positivo ( 3 ítems, por ejemplo, «Mi jefe inmediato siempre piensa en positivo», prudencia y perseverancia ( 3 ítems, por ejemplo, «Mi jefe inmediato es prudente actuando de forma justa, adecuada y con cautela»; e integridad (3 ítems, por ejemplo, «Mi jefe inmediato aprende de sus errores preocupándose por su superación personal».

El cuestionario de cultura organizacional compuesto por cuatro dimensiones: implicación (3 ítems, por ejemplo, « En mi empresa cuento con la autoridad, iniciativa y la capacidad para 
realizar mi trabajo »), consistencia (5 ítems, por ejemplo, «En mi empresa, los empleados no tienen limitaciones para la realización de su trabajo», adaptabilidad (4 ítems, por ejemplo, «Mi empresa trata de aprender para responder a los cambios del entorno»; y misión (3 ítems, por ejemplo, «En mi empresa los empleados participan en la dirección estratégica para mantener los objetivos organizacionales». En ambos cuestionarios la escala de respuesta con categoría tipo Likert oscilaba de 1 («Completamente falso») a 5 («Completamente verdadero»).

El procesamiento de los datos se llevó a cabo a través del software SPSS versión 22 para obtener los resultados del estudio.

\section{Resultados}

Los resultados enunciaron que en la evaluación del coeficiente de confiabilidad el procesamiento de los datos arrojó en sus 18 ítems el coeficiente de 0,96 de Alfa de Cronbach para el cuestionario de liderazgo ético y en sus 15 ítems el coeficiente de 0,85 de Alfa de Cronbach para el cuestionario de cultura organizacional. Del mismo modo, al identificar los rasgos de la personalidad de los líderes éticos en las pymes de Imbabura, la Figura 1 distingue que todos los rasgos que caracterizan los líderes éticos están presentes en las pymes en Imbabura.

Los hallazgos de la investigación enuncian que el $85 \%$ de los empleados considera que su líder considerado y reflexivo, es una persona que asume sus compromisos (Rodríguez, 2008), piensa en el bienestar de los demás, es humilde, honesto, no discrimina sus empleados (Mihelic et al., 2010) y cumple lo que promete respetando sus derechos (Gómez, 2006). El 81\% de los encuestados señalan que su líder es bondadoso y respetuoso con sus subordinados al escuchar sus problemas o las quejas buscando la orientación de los empleados hacia el bienestar común

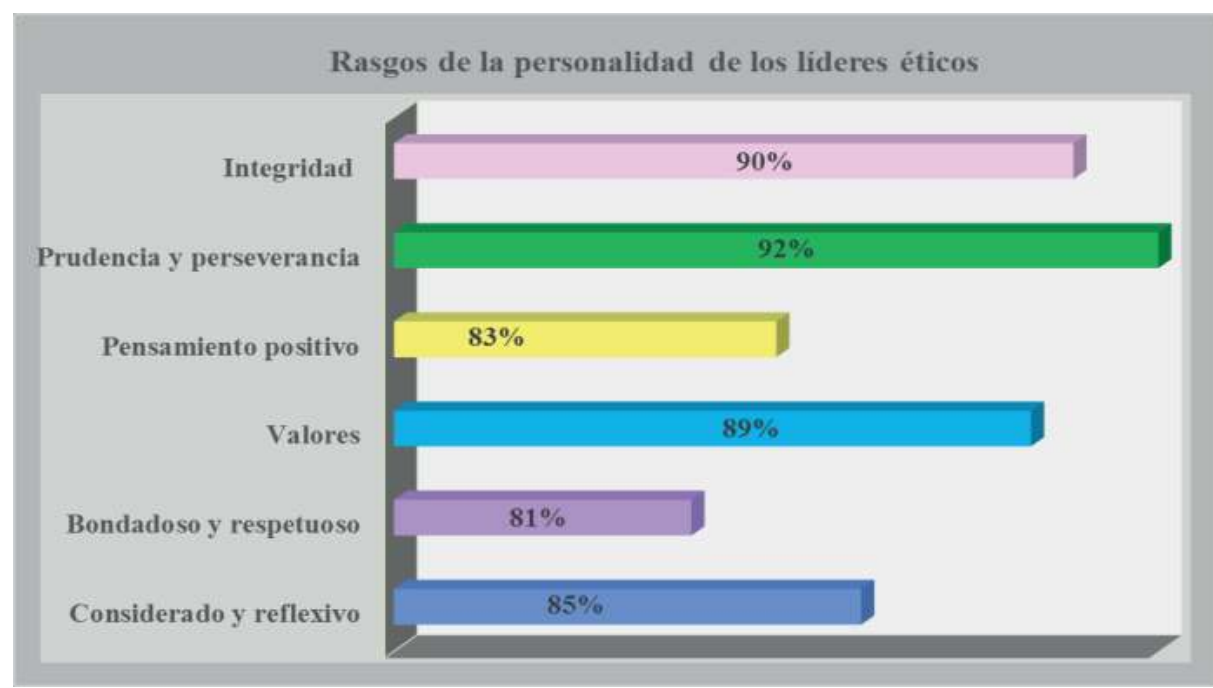

Figura 1. Personalidad del liderazgo ético.

Fuente: Elaboración propia. 
(Cortina, 2007). El 89\% de los empleados arguye que los líderes de las empresas poseen valores de honestidad, respeto, solidaridad, tolerancia y flexibilidad (Gómez, 2006). El 83\% afirma que los líderes mantienen un pensamiento positivo sobre lo que es bueno para la empresa y el desarrollo de los empleados. El 92\% de los empleados afirman que los líderes son prudentes y perseverantes, actúan en forma justa, son cautelosos y controlan sus emociones (Soto y Cárdenas, 2007). El 90\% de los sujetos encuestados señala que los líderes de las pymes muestran integridad, aprenden de sus errores y siempre tratan de superarse (Mihelic et al., 2010), fomentan el trabajo en equipo (Frondizi, 2005) creando un ambiente sano para la productividad de la organización (Pacheco y Sierra, 2007).

En el mismo orden de ideas, vale destacar que al conocer las características de la cultura organizacional en las pymes de Imbabura, la Figura 2 refleja que el $75 \%$ de los sujetos encuestados afirma la presencia de la implicación como característica de la cultura organizacional denotando que cuenta con autoridad, iniciativa y capacidad para realizar su trabajo, señala que en su empresa se valora el trabajo cooperativo debido a que la gerencia fomenta el desarrollo de las capacidades de los trabajadores a través de la inversión para mantener la competitividad (Denison et al., 2012).

Del mismo modo, el $90 \%$ de los sujetos encuestados afirma que en las pymes se comparten los valores fundamentales creando un sentido de identidad $y$ un conjunto claro de expectativas. Cuando existen desacuerdos se trata de encontrar soluciones en las que todos puedan ganar, se coordina la integración de las diferentes funciones y unidades de la organización de tal manera que los empleados no tengan limitaciones para la realización de sus tareas para que exista la consistencia de la cultura organizacional (Denison et al., 2012). El

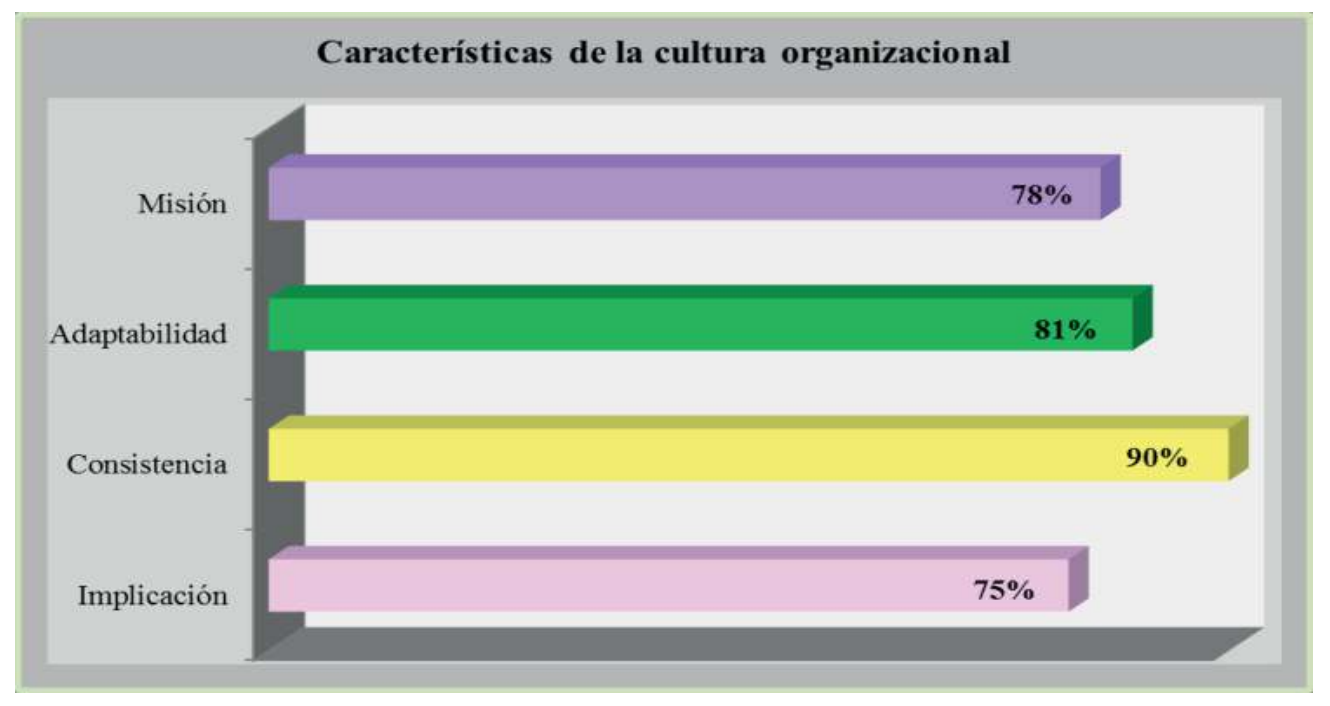

Figura 2. Cultura organizacional.

Fuente: Elaboración propia. 
$81 \%$ de los empleados considera que las pymes se caracterizan por la adaptabilidad reflejada en su disponibilidad para responder a los cambios del entorno (Denison et al., 2003), brindando a los empleados la posibilidad de aprender nuevas habilidades para comprender los clientes y fomentando el aprendizaje organizacional a través de la innovación y el desarrollo de las capacidades de los trabajadores (Denison et al., 2012). El $78 \%$ de los empleados acota que misión está presente en las empresas, los empleados participan en la dirección estratégica para mantener los objetivos organizacionales. Se distinguen claramente las metas y los objetivos que vinculan la misión, la visión y la estrategia de la organización y se percibe la visión compartida entre los empleados encarnada por los valores fundamentales brinda orientación y dirección (Denison et al., 2012).

En la Tabla 1 se presenta la matriz de correlaciones entre variables y dimensiones del liderazgo ético y la cultura organizacional en las pymes de Imbabura. Se observan correlaciones positivas muy fuertes entre las dimensiones del liderazgo ético y las dimensiones de la cultura organizacional.

Entre el liderazgo ético y la cultura organizacional existe un coeficiente de correlación de Spearman de 0,837, señalando una relación positiva, fuerte y estadísticamente significativa, es decir que a mayor presencia de los rasgos de la personalidad de liderazgo auténtico en los líderes, mayor presencia de las características de la cultura organizacional de las pymes de Imbabura.

Tabla 1.

Correlaciones entre las variables y las dimensiones

\begin{tabular}{|c|c|c|c|c|c|c|c|c|c|c|c|c|}
\hline & Cyr & Byr & Val & $\mathrm{Pp}$ & Pyp & Int & LE & $\operatorname{Imp}$ & Cons & Adap & Mis & $\mathrm{CO}$ \\
\hline Cyr & 1 & & & & & & & & & & & \\
\hline Byr & $1,000^{* *}$ & 1 & & & & & & & & & & \\
\hline Val & $1,000^{* *}$ & $1,000^{* *}$ & 1 & & & & & & & & & \\
\hline $\mathrm{Pp}$ & $1,000^{* *}$ & $1,000^{* *}$ & $1,000^{* *}$ & 1 & & & & & & & & \\
\hline Pyp & $1,000^{* * *}$ & $1,000^{* *}$ & $1,000^{* *}$ & $1,000^{* *}$ & 1 & & & & & & & \\
\hline Int & $1,000^{* *}$ & $1,000^{* *}$ & $1,000^{* *}$ & $1,000^{* *}$ & $1,000^{* *}$ & 1 & & & & & & \\
\hline LE & $1,000^{* *}$ & $1,000^{* *}$ & $1,000^{* *}$ & $1,000^{* *}$ & $1,000^{* *}$ & $1,000^{* *}$ & 1 & & & & & \\
\hline $\operatorname{Imp}$ &, $990^{* *}$ &, $990^{* *}$ &, $990^{* *}$ &, $990^{* *}$ &, $990^{* *}$ &, $990^{* *}$ &, $990^{* *}$ & 1 & & & & \\
\hline Cons & $837^{* *}$ & $837^{* *}$ & $837^{* *}$ & $837^{* *}$ & $837^{* *}$ & $837^{* *}$ & $837^{* *}$ &, $886^{* *}$ & 1 & & & \\
\hline Adap & $827^{* *}$ &, $827^{* *}$ & $827^{* *}$ &, $827^{* *}$ &, $827^{* *}$ &, $827^{* *}$ &, $827^{* *}$ &, $876^{* *}$ &, $991^{* *}$ & 1 & & \\
\hline Mis &, $827^{* *}$ &, $827^{* *}$ & $827^{* *}$ &, $827^{* *}$ & $827^{* *}$ &, $827^{* *}$ & $827^{* *}$ &, $876^{* *}$ &, $991^{* *}$ & $1,000^{* *}$ & 1 & \\
\hline $\mathrm{CO}$ & $837^{* *}$ &, $837^{* *}$ & $837^{* *}$ &, $837^{* *}$ & $837^{* *}$ &, $837^{* *}$ & $837^{* *}$ &, $886^{* *}$ & $1,000^{* *}$ &, $991^{* *}$ &, $991^{* *}$ & 1 \\
\hline
\end{tabular}

Fuente: Elaborado a base del resultado de la investigación

Nota: **. La correlación es significativa en el nivel 0,01 (bilateral).

Nota: Cyr: Considerado y reflexivo, Byr: Bondadoso y respetuoso, Val: valores, Pp: Pensamiento positivo, Pyp: Prudencia y perseverancia, Int: integridad, LE: Liderazgo Ético, Imp: implicación, Cons: Consistencia, Adap: Adaptabilidad, Mis: Misión, CO: Cultura Organizacional 
Estos hallazgos confirman la relación entre el liderazgo y la cultura ya que uno requiere del otro (Schein, 2010). Los líderes fundadores de las pymes se preocupan por mantener una buena comunicación con los trabajadores (Fairholm, 1994) y cuando la organización se encuentra en un período de crisis (Trice y Beyer, 1993). La presencia de la ética en estos líderes en su trabajo diario respalda los principios éticos y las normas como fundamento para orientar la cultura organizacional (Mintzberg, 2002).

Los resultados demostraron que los empleados de las pymes perciben en los líderes de las pymes los rasgos de la personalidad del liderazgo ético y que éstos rasgos tienen una correlación positiva y directamente proporcional sobre las características de la cultura organizacional. En este sentido, los hallazgos revelaron que los líderes de las pymes luchan por la equidad y la justicia sin discriminación, controlan su autoridad, son servidores con valores éticos desempeñando sus funciones de manera cordial para alcanzar sus metas y muestran su responsabilidad hacia sus compromisos reflejando su honestidad. Además, el análisis correlacional mostró que el comportamiento de los líderes fortalece la cultura organizacional: (i) en apoyo del empoderamiento de los empleados impulsando la capacidad para administrar su propio trabajo y estimulando el compromiso personal, (ii) en coordinar e integrar las actividades con valores fundamentales hacia el logro de los objetivos generando un sentido de identidad en los empleados, (iii) en la creación de cambios y formas para asumir las transformaciones producidas por el veriginoso crecimiento de la globalización, y (iv) en el fomento de una visión compartida de la misión y los objetivos estrategicos de la oganización.

\section{Conclusiones}

Los resultados de la investigacion permiten inferir que en las pymes de Imbabura los empleados perciben los rasgos de la personalidad de los líderes éticos manifestando que los supervisores de las empresas son personas consideradas y reflexivas, bondadosas y respetuosas, poseen valores y principios éticos, un pensamiento positivo, son prudentes y perseverantes y fomentan la integridad en el trabajo.

Asimismo, se infiere que los empleados de las empresas estudiadas permiten evidenciar los rasgos o pilares que caracterizan la cultura organizacional dentro de las organizaciones. Las pymes se caracterizan por su rasgo de implicación en el cual los empleados disfrutan del empoderamiento, su orientación hacia el trabajo en equipo y del desarrollo de sus capacidades. La consistencia se infiere por la aceptación de los empleados de compartir los valores fundamentales y de llegar a acuerdos para solventar situaciones en función de las actividades de coordinación e integración que les brinda la organización. La adaptabilidad en la capacidad de las organizaciones para crear cambios enfocados a la satisfacción de las necesidades de los clientes y en el fomento de la innovación a través del 
aprendizaje organizacional; y la misión en las estrategias gerenciales para alcanzar las metas y los objetivos de las empresas enmarcados en una visión compartida.

Finalmente, se concluye que existe una correlación directa, alta, $\mathrm{y}$ significativa entre los rasgos de la personalidad de los líderes éticos y las características de la cultura organizacional en las pequeñas y medianas empresas de Imbabura que permite inferir que en la medida en que se incrementan los rasgos de la personalidad del liderazgo ético, en esa misma medida, se incrementan las características de la cultura organizacional. Por lo tanto, se infiere que los rasgos de la personalidad del liderazgo ético permiten el fortalecimiento de una cultura organizacional en las pymes de Imbabura.

La investigación proporciona nuevos conocimientos sobre las relaciones entre el liderazgo ético y la cultura organizacional, pero se limitó a una ciudad de Ecuador. Se sugiere a partir de estos hallazgos, la realización de futuras investigaciones que contemplen en primer lugar, hallazgos de otras ciudades y de otros países para comparar los resultados. En segundo lugar, una variable mediadora como el compromiso laboral, el comportamiento laboral innovador o el compromiso con el proceso innovador en los empleados para ampliar el conocimiento.

\section{Contribución de autores}

M.S.L.P. Idea, revisión de literatura, metodología, análisis de datos, discusión y conclusiones conjuntas, revisión de redacción.

B.P.A.I. Revisión de literatura, metodología, discusión y conclusiones conjuntas, revisión de redacción.

\section{Referencias}

Ahmad, I., y Gao, Y. (2018). Ethical leadership and work engagement: The roles of psychological empowerment and power distance orientation. Management Decision, 56(9), 1991-2005.

Aktouf, O. (2002). Administración y pedagogía. Medellín: Fondo Editorial Universidad Eafit.

Alshammari, A., N. Almutairi, N., y Fahad Thuwaini, S. (2015). Ethical Leadership: The Effect on Employees. International Journal of Business and Management, 10(3). doi:10.5539/ijbm.v10n3p108

Arias, F. (2012). El proyecto de investigación. Introducción a la metodología científica. Venezuela: Episteme.

Avolio, B., y Bass, B. (1995). Individual consideration viewed at multiple levels of analysis: A multi-level framework for examining the diffusion of transformational leadership. The Leadership Quarterly, 6(2), 199-218.

Avolio, B., y Gardner, W. (2005). Authentic leadership development: Getting to the root of positive forms of leadership. The Leadership Quarterly, 16(3), 315-338.

Banks, G. C., Fischer, T., Gooty, J., y Stock, G. (2020). Ethical leadership: Mapping the terrain for concept cleanup and a future research agenda. The Leadership Quarterly, 32(2), 1-14.

Bañón, M. (2007). La ética en la gestión empresarial. Mc Graw Hill. 
Bedi, A., Alpaslan, C., y Green, S. (2016). A metaanalytic review of ethical leadership outcomes and moderators. Journal of Business Ethics, 139(3), 517-536.

Bernal, C. (2010). Metodología de la investigación. administración, economía, humanidades $y$ ciencias sociales. Tercera Edición. Pearson Educación.

Brown, M., y Treviño, L. (2006). Ethical leadership: A review and future directions. The Leadership Quarterly, 17(6), 595-616.

Brown, M., Treviño, L., y Harrison, D. (2005). Ethical leadership: A social learning perspective for construct development and testing. Organizational Behavior and Human Decision Processes, 97(2), 117-134.

Burke, W., y Litwin, H. (1992). A causal model of organizational performance and change. Journal of Management, 18(3), 523-545.

Cameron, K., y Quinn, R. (2011). Diagnosing and changing organizacional culture: Based on the Competing Values Framework (3. ${ }^{\text {a }}$ ed.). Jossey-Bass.

Cardozo, E., Velásquez, Y., y Rodríguez, C. (2012). Revisión de la definición de PYME en América Latina. Global Conference on Business and Finance Proceedings, 7(2), 1657-1668.

Chiavenato, I. (2009). Comportamiento Organizacional: La dinámica del éxito en las organizaciones. Segunda Edición. McGraw Hill.

Correa, J., Rodríguez, M., y Alonso, M. (2018). Liderazgo ético en las organizaciones: una revisión de la literatura. AD-minister, (32), 57 - 82. https://doi.org/10.17230/administer.32.3.

Cortina, A. (2007). El Mundo de los Valores: "ética mínima y educación”. 6ta Edición. Editorial El Búho.
Creswell, J., y Creswell, J. (2018). Research design: qualitative, quantitative, and mixed methods approaches. Fifth edition. SAGE Publications.

Daft, R. (2015). Teoría y diseño organizacional. Cengage Learning Editores.

Davis, K., y Newstrom, J. (2001). Comportamiento Humano en el Trabajo. 10ma. Edición. McGraw Hill.

Denison, D. (1996). What is the difference between organizational culture and organizational climate? A native's point of view on a decade of paradigm wars. Academy of Management Review, 21(3), 619-654.

Denison, D., Haaland, S., y Goelzer, P. (2003). Corporate culture and organizational effectiveness: Is Asia different From the rest of the world? En J. Osland, Advances in Global Leadership, 3 (págs. 205-227). West Yorkshire: Emerald GroupPublishing Limited.

Denison, D., Hooijberg, R., Lane, N., y Lief, C. (2012). Leading Culture change in global organizations. Alining culture and strategy. Jossey-Bass.

Denison, D., Nieminen, L., y Lindsey, K. (2012). Diagnosing organizational cultures: A conceptual and empirical review of culture effectiveness surveys. European Journal of Work and Organizational Psychology, 23(1), 1-17.

Donawa, Z. (2019). Liderazgo ético en la gerencia de las empresas del sector eléctrico del estado Zulia de Venezuela. Impacto Cientifico, 14(1), 59-81.

Enderica, O., D'armas, M., Bermeo, R., Lopez M., Tinoco, E., y Carrión, G. (2018). Buen liderazgo, una experiencia en el crecimiento empresarial de las PYME. Espacios, 39(42), 4.

Espinoza-Solís, E., y Elgoibar, P. (2019). Leadership 
patterns en Ecuadorian managers: The impact of gender and education. Inclusiones, 6(11), 178-197.

Fairholm, G. (1994). Leadership and the Culture of Trust. Praeger Publishers.

Frondizi, R. (2005). ¿Qué son los valores? Introducción a la axiología. Fondo de Cultura Económica.

Gómez, E. (2006). El Liderazgo ético: un desafio de nuestro tiempo. Gestión 2000.

Hernández, R., Fernández, C., y Baptista, M. (2014). Metodología de la investigación. Sexta edición. McGraw-Hill.

Instituto Nacional de Estadística y Censos - INEC. (2018). Boletín, los resultados del Directorio de Empresas y Establecimientos (DIEE) correspondiente al periodo 2018. Quito, Ecuador.

Jiménez, W., Pazmay, P., y Mancheno, M. (2017). Impacto de la cultura organizacional al implementar un modelo de gestión basado en la metodología del plan de negocios como factor clave para la innovación de las Pymes. Publicando, 4(12), 315-333.

Joplin, T., Greenbaum, R. L., Wallace, J. C., y Edwards, B. D. (2021). Employee entitlement, engagement, and performance: The moderating effect of ethical leadership. Journal of Business Ethics, 168(1), 1-14.

Kanungo, R. (2001). Ethical values of transactional and transformational leaders. Canadian Journal of Administrative Sciences, 18(4), 257-265.

Khuntia, R., y Suar, D. (2004). A scale to assess ethical leadership of Indian private and public sector managers. Journal of Business Ethics, 49(1), 13-26.

Khuong, M. N., y Nhu, N. V. Q. (2015). The Effects of Ethical Leadership and Organizational Culture towards Employees' Socialibility and Commitment: A Study of Tourism Sector in Ho Chi Minh City, Vietnam. Journal of Advanced Management Science, 3(4), 329 - 336.

Lam, L., Nguyen, P., Le, N., y Tran, K. (2021). The relation among organizational culture, knowledge management, and innovation capability: Its implication for open innovation. Journal of Open Innovation: Technology, Market, and Complexity, 7(1:66), 1-16.

Leal Paredes, S. Salomon, J., y Rivera Camino, J. (2021). Impact of Authentic Leadership on Work Engagement and Organizational Citizenship Behavior: The Meditation Role of Motivation for Work, International Journal of Economics \& Business Administration, 9(3), 3-31. DOI: $10.35808 / \mathrm{ijeba} / 716$

Lindblom, A., Kajalo, S., y Mitronen, L. (2015). Exploring the links between ethical leadership, customer orientation and employee outcomes in the context of retailing. Management Decision, 53(7), 1642-1658.

Llanos, M. (2018). La cultura organizacional, principal propulsor de la calidad en instituciones de educación superior: caso universidad ecuatoriana. Espacios, 39(23), 29.

Luthans, F. (2011). Organizational behavior: an evidence-based approach. Twelfth Edition. McGraw-Hill .

Méndez, C. (2012). Metodología : diseño y desarrollo del proceso de investigación con énfasis en ciencias empresariales. 4 ed. Limusa.

Mihelic, K., Lipicnik, B., y Tekavcic, M. (2010). Ethical Leadership. International Journal of Management and Information Systems, 14(5), 31-42. 
Mintzberg, H. (2002). El proceso estratégico. Prentice Hall.

Molero, F. (2002). Cultura y liderazgo una relacion multifacética. Boletin de psicología, (76), $53-75$.

Newstrom, J. (2011). Comportamiento humano en el trabajo. Decimotercera edición. McGraw-Hill.

Pacheco, M., y Sierra, M. (2007). Educación y valores. Limusa.

Palafox Soto, M. O., Ochoa Jiménez, S., y Jacobo Hernández, C. A. (2021). Leadership and its relationship with the organizational culture in small and medium sized family businesses in Ciudad Obregon, Sonora. Nova Scientia, 13(26). https://doi.org/10.21640/ns.v13i26.2682

Rodríguez, M. (2008). Comportamiento Ético Gerencial. Bogotá: Universidad Nacional de Colombia.

Schein, E. (2004). Organizational Culture and Leadership. 3rd Edition. Jossey-Bass.

Schein, E. (2010). Organizational culture and leadership, 4th edition. Jossey-Bass.

Sierra, R. (2000). Técnicas de Investigación Social. 5ta Edición. Paraninfo.

Soto, E., y Cárdenas, J. (2007). Ética en las Organizaciones. Mc Graw Hill.

Trice, H., y Beyer, J. (1993). The cultures of work organization. Prentice Hall.

Yukl, G. (2010). Leadership in Organizations (7.a ed.). Prentice Hall. 
